\title{
Durabilidade natural de cinco espécies madeireiras da Caatinga em ensaio de deterioração em campo aberto e natural
}

\author{
Líllia Kaliany Campos Carlos $\mathbb{(}^{1}$ Marco Antonio Diodato $\mathbb{( 1}^{1}$ Vinicius Gomes Castro (ํ)
}

${ }^{1}$ Universidade Federal Rural do Semiárido, Av. Francisco Mota, 572, Pres. Costa e Silva, Mossoró, RN, Brasil

\section{Original Article \\ *Corresponding author: vinicius.castro@ufersa.edu.b $\mathrm{r}$ \\ Palavras-chave: \\ Campo de apodrecimento \\ Índice de deterioração \\ Perda de massa}

Keywords:

Rotten field

Deterioration Index

Weight loss

Received in

2021/06/17

Accepted on

2021/09/21

Published in

2021/10/11

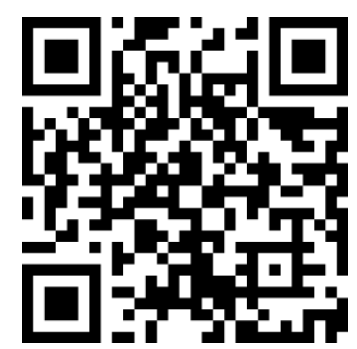

DOI:

https://doi.org/10.34062/afs. v8i3.12631
RESUMO: Uma forma de viabilizar o investimento em planos de manejo florestal e reduzir a devastação da Caatinga é a comercialização de produtos madeireiros de maior valor agregado por comunidade rurais locais. Para que isso se torna uma realidade é necessário investigar e caracterizar as diversas espécies madeireiras nativas para que as de maior potencial sejam sugeridas. Desta forma, este trabalho teve como objetivo determinar a durabilidade natural de cinco espécies da Caatinga de densidade de moderadamente alta a alta (entre $0,550 \mathrm{~g} / \mathrm{cm}^{3}$ e 0,950 $\mathrm{g} / \mathrm{cm}^{3}$ ). Foram instalados dois campos de apodrecimento: a) aberto, sem vegetação ao redor; b) natural, em área de fragmento de Caatinga. Em cada campo, foram colocadas estacas de $2 \times 2 \times 30 \mathrm{~cm}$, enterradas com $15 \mathrm{~cm}$ de profundidade, das espécies Angico (Anadenanthera colubrina (Vell) Brenan)), Aroeira (Myracrodruon urundeuva (Allemao)), Jurema Preta (Mimosa tenuiflora (Mart) Benth)), Pau Branco (Auxemma oncocalyx (Allemao) Taub.)) e Pereiro (Aspidosperma pyrifolium Mart). Os campos foram instalados entre fevereiro e março de 2019. As estacas tiveram a perda de massa e o índice de deterioração determinados após 90, 200 e 270 dias. Todas as espécies obtiveram perda de massa abaixo de $10 \%$ após o fim do período do experimento, o que as classificou como altamente resistente. A maior parte da deterioração ocorreu nos primeiro 90 dias, que coincidiu com o período chuvoso da região. As espécies Anadenanthera colubrina e Myracrodruon urundeuva, além do ataque por fungos, também foram deterioradas por cupins do gênero Heterotermes e por coleópteras.

\section{Natural durability of five wood species from Caatinga in deterioration tests in open and natural fields}

\begin{abstract}
One way to make viable forest management plans and reduce the Caatinga devastation is the commercialization of wood products with higher added values by local rural communities. For that to happen, it is necessary to research and characterize the several native wood species to be able to suggest the best potential options. Therefore, this work aimed to appoint the natural durability of five species native to Caatinga with moderated high to high density (between $0.550 \mathrm{~g} / \mathrm{cm}^{3}$ and $0.950 \mathrm{~g} / \mathrm{cm}^{3}$ ). Two rotten fields were implemented: a) open, without close vegetation; b) natural, within a Caatinga fragment. Into each field, Angico (Anadenanthera colubrina (Vell) Brenan)), Aroeira (Myracrodruon urundeuva (Allemao)), Jurema Preta (Mimosa tenuiflora (Mart) Benth)), Pau Branco (Auxemma oncocalyx (Allemao) Taub.)) and Pereiro (Aspidosperma pyrifolium Mart) $2 \times 2 \times 30 \mathrm{~cm}$ stakes, buried to $15 \mathrm{~cm}$, were placed. The fields were installed between February and March of 2019 and the stakes had their weight loss and deterioration index determined after 90, 200 and 270 days. All species showed weigh loss under $10 \%$ after the total experiment time and were classified as highly resistant. Most of the deterioration happened in the first 90 days, that period matched with the local rainy season. Anadenanthera colubrina and Myracrodruon urundeuva species, besides the fungi attack, was also attacked by termites from the Heterotermes genre and by beetles.
\end{abstract}




\section{Introdução}

A Caatinga é o único bioma exclusivamente brasileiro e possui área de vegetação nativa de aproximadamente 32 milhões de hectares, que representa cerca de metade da sua cobertura original. Porém, a vegetação remanescente ainda está potencialmente em risco, principalmente por sua alta fragmentação. Na Caatinga, $75 \%$ da vegetação está a menos de $1 \mathrm{~km}$ da área limítrofe destes fragmentos e esta proporção sobe para $99 \%$ se considerar a distância deste limite como $5 \mathrm{~km}$ (Antongiovanni et al. 2020). Essa perigosa relação entre devastação e região de borda dos fragmentos deve-se ao fato das espécies madeireiras serem amplamente utilizadas como matéria prima pelas comunidades rurais da região, principalmente no que se refere à geração de energia. De acordo com Lima et al. (2018), é justamente o uso de material lenhoso como combustível que apresenta um dos maiores potenciais destrutivos, que se torna ainda mais crítico quando considerada a dinâmica de renovação do material.

Lima et al. (2018) apontaram que o uso de combustível madeireiro por comunidades rurais geralmente é uma segunda opção quando se torna necessário economizar dinheiro devido ao custo do gás de cozinha. Lenha geralmente é uma matéria prima acessível devido a informalidade do mercado que oferta material ilegal a baixos preços. Porém, os custos de produção tendem a subir e, consequentemente, os seus preços, caso o sistema de exploração deixasse de ser manual (com machado e foice) e se considerar a exigência legal de planos de manejo (Lopes e Canto, 2018). Desta forma, a região enfrenta um dilema: o baixo preço da lenha não gera lucro suficiente para justificar o investimento necessário para se fazer um plano de manejo.

Uma possível solução para esse empasse do desenvolvimento econômico das comunidades rurais da região Nordeste e a preservação do bioma Caatinga seria o manejo sustentável com foco na exploração de um produto madeireiro com maior valor agregado do que a lenha. Contudo, antes que uma espécie madeireira possa ser sugerida como matéria-prima para produtos nobres, é necessário que a madeira seja caracterizada tecnologicamente para assegurar que irá cumprir com os requisitos mínimos solicitados. Dentre as propriedades que devem ser avaliadas, a durabilidade natural é essencial, principalmente para uso na construção civil e indústria moveleira. Conhecer a vida útil do seu material poupa gastos desnecessários com substituição de peças e reduz impactos ao meio ambiente, uma vez que pode orientar a não utilização de preservantes químicos (Carvalho e Loiola, 2018).

Dentre as inúmeras espécies do bioma Caatinga com potencial madeireiro, cinco podem ser destacadas. A madeira da espécie Anadenanthera colubrina (Vell) Brenan), conhecida popularmente como Angico, é tradicionalmente usada na construção civil e na produção de cercas e lenha pelas comunidades locais. Sendo que o uso como mourão justificado pela sua densidade e resistência ao ataque de cupins. A durabilidade de mourões desta espécie é relatada como de no mínimo 5 anos de uso (Monteiro et al., 2006). A madeira de Aroeira (Myracrodruon urundeuva (Allemao)) também é amplamente utilizada na construção civil e como mourão, por ser considerada resistente ao apodrecimento e ataque de cupins de madeira seca quando utilizada em contato com o chão (Lucena et al., 2011). Por sua vez, a madeira de Jurema Preta (Mimosa tenuiflora (Mart) Benth)) é amplamente utilizada na região da Caatinga, sendo a espécie nativa mais transportada no estado do Rio Grande do Norte. Seu uso atual é principalmente na produção de energia, embora também haja registros de seu uso como estacas e moirões (Sousa Júnior, Canto e Costa. 2021). Já a espécie Pau Branco (Auxemma oncocalyx (Allemao) Taub.)) é abundante na Caatinga, chegando a ser relatado 4200 indivíduos/ha (Ivanov et al. 2019), e apresenta uma madeira moderadamente pesada e de boa resistência mecânica (Souza et al. 2020). E por último, a espécie Aspidosperma pyrifolium Mart, conhecida como Pereiro, considerada uma das mais frequentes na região do Seridó, RN (Amorim, Sampaio e Araújo, 2009). Chaves et al. (2014) apontaram a madeira desta espécie como uma das mais utilizadas na produção de cercas no estado do Piauí.

Contudo,a madeira é um material orgânico e como tal está propenso ao ataque de agentes deterioradores. Fungos e insetos fazem parte das duas principais categorias de agentes bióticos que podem buscar sua fonte de energia nos polissacarídeos formadores da parede celular. Este ataque resulta no apodrecimento, descoloração ou danos mecânicos na madeira que afeta diretamente o preço e funcionalidade da madeira. A durabilidade natural de uma madeira pode ser compreendida como sua capacidade de resistir, em diferentes graus, a este ataque. Carvalho, Santini e Gouveia (2015) atribuíram a diferença de suscetibilidade à deterioração de cada tipo de madeira a sua diferente característica anatômica e composição química.

O ideal é que a durabilidade natural seja avaliada em ensaios de campo de apodrecimento implantados em ambiente livre de vegetação, caracterizado como campo de apodrecimento aberto, e em ambiente natural com ampla vegetação. Ambos reproduzem com fidelidade situações e microrganismos os quais os produtos derivados destas madeiras possam vir a ser expostos, principalmente quando estes forem utilizados em contato direto com o solo (Mattos et al., 2013).

Diante disto, o objetivo deste estudo foi avaliar a durabilidade natural em campo de apodrecimento aberto e natural de madeiras de cinco espécies nativas do bioma Caatinga: Angico 
(Anadenanthera colubrina); Aroeira (Myracrodruon urundeuva); Jurema Preta (Mimosa tenuiflora); Pau Branco (Auxemma oncocalyx); e Pereiro (Aspidosperma pyrifolium).

\section{Material e Métodos}

Foram produzidas estacas com dimensões de $2 \times 2 \times 30$ $\mathrm{cm}$, isentas de defeitos, de madeiras de cinco diferentes espécies retiradas de uma área sob plano de manejo florestal localizada no município de Upanema, RN. As espécies avaliadas foram Anadenanthera colubrina, Aspidosperma pyrifolium, Auxemma oncocalyx, Mimosa tenuiflora e Myracrodruon urundeuva. Todas as espécies selecionadas possuem madeiras consideradas de moderadamente pesadas (densidade entre 0,550 e $0,750 \mathrm{~g} / \mathrm{cm}^{3}$ ) a pesadas (densidade entre $0,750 \mathrm{e}$ $0,950 \mathrm{~g} / \mathrm{cm}^{3}$ ), de acordo com classificação sugerida por Eleotério et al. (2015).

Tabela 1. Dados médios da climatologia para o município de Mossoró- RN referentes aos meses de fevereiro a dezembro de 2019

\begin{tabular}{ccc}
\hline Meses & Umidade Relativa do ar média (\%) & Precipitação (mm) \\
\hline Fevereiro & 76,4 & 100,232 \\
Março & 86,4 & 264,664 \\
Abril & 86,7 & 225,044 \\
Maio & 83,1 & 53,342 \\
Junho & 74,5 & 25,396 \\
Julho & 70,0 & 2,794 \\
Agosto & 61,4 & 0,254 \\
Setembro & 62,6 & 0 \\
Outubro & 61,8 & 0,254 \\
Novembro & 65,3 & 0 \\
Dezembro & 64,7 & 11,682 \\
\hline
\end{tabular}

Foi determinada a perda de massa das amostras através da diferença entre peso seco inicial e o peso determinado após 90, 200 e 270 dias de exposição. Para realização das análises, foi feito sorteio de forma aleatória para retirada de uma estaca de cada espécie por bloco. As estacas não retornaram ao campo após serem secas para determinação da perda de massa.

Também foi realizada a análise visual do índice de deterioração das amostras sugerido por Lepage (1986) (Tabela 2). Por se tratar de um método subjetivo, essas notas foram atribuídas individualmente por três observadores diferentes.
Para cada espécie, foram produzidos 18 corpos de prova marcados e pesados para determinação da massa seca e densidade básica. As estacas foram divididas igualmente e instaladas em dois campos de apodrecimento montados em área

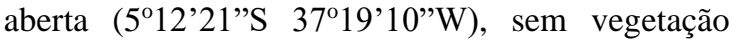
próxima, e em área de Caatinga nativa (5'12'23"S 37¹9'16”W), ambos localizados no município de Mossoró, RN. Em cada campo, as amostras foram distribuídas aleatoriamente em três blocos com três repetições de cada espécie por bloco.

As estacas foram enterradas até $15 \mathrm{~cm}$ de seu comprimento, ficando os outros $15 \mathrm{~cm}$ expostos. Foi estabelecido um espaçamento de $25 \mathrm{~cm}$ entre estacas e de $1 \mathrm{~m}$ entre blocos. As amostras ficaram no campo por 270 dias, entre fevereiro e dezembro de 2019. O experimento foi instalado durante o período chuvoso (Tabela 1).

Tabela 2. Classificação do nível de deterioração da madeira sugerido por Lepage (1986)

\section{Estado de sanidade}

Sadio- nenhum ataque

Ataque leve ou moderado de fungos ou de térmitas

Ataque evidente, mas moderado de fungos e térmitas

Apodrecimento intenso ou ataque interno de térmitas

Quebra/ perda quase total de resistência
Durante o período de análise, quando se observou a presença de insetos, estes foram coletados e acondicionados em frasco fechado com álcool $70 \%$ e identificados em laboratório através da chave proposta por Constantino (1999).

Foi realizada análise de variância para verificar o efeito da espécie e do campo de apodrecimento na perda de massa do material. Na verificação de existência de diferença estatística entre médias, foi aplicado o teste de Tukey a 5\% de probabilidade de erro. 


\section{Carlos et al.}

\section{Resultados}

$\mathrm{Na}$ tabela 3, foram apresentados os de perda de massa para as espécies da Caatinga com densidade básica entre 0,665 e $0,909 \mathrm{~g} / \mathrm{cm}^{3}$ em campo de apodrecimento aberto. Sendo que não houve diferença estatística entre os valores médios de densidade básica das madeiras de Anadenanthera columbrina $(0,909 \mathrm{~g} / \mathrm{cm} 3)$ e Myracrodrum

Tabela 3. Valores de perda de massa (\%) e densidade básica para as cinco espécies da Caatinga submetidas ao campo de apodrecimento aberto após 90, 200 e 270 dias de exposição.

\begin{tabular}{ccccc}
\hline Espécies & $\begin{array}{c}\text { Densidade } \\
\text { Básica }\left(\mathbf{g} / \mathbf{c m}^{\mathbf{3}}\right)\end{array}$ & $\mathbf{9 0}$ dias & $\mathbf{2 0 0}$ dias & $\mathbf{2 7 0 ~ d i a s}$ \\
\hline Anadenanthera colubrina & $0,909 \mathrm{D}$ & $2,12 \mathrm{~A} \mathrm{ab}$ & $2,05 \mathrm{~A} \mathrm{a}$ & $3,65 \mathrm{~B} \mathrm{~b}$ \\
Aspidosperma pyrifolium & $0,665 \mathrm{~A}$ & $2,04 \mathrm{~A} \mathrm{a}$ & $1,76 \mathrm{~A} \mathrm{a}$ & $1,35 \mathrm{~A} \mathrm{a}$ \\
Auxemma oncocalyx & $0,687 \mathrm{AB}$ & $1,63 \mathrm{~A} \mathrm{a}$ & $2,39 \mathrm{~A} \mathrm{a}$ & $1,80 \mathrm{AB} \mathrm{a}$ \\
Mimosa tenuiflora & $0,779 \mathrm{BC}$ & $1,29 \mathrm{~A} \mathrm{a}$ & $2,24 \mathrm{~A} \mathrm{a}$ & $2,08 \mathrm{AB}$ a \\
Myracrodruon urundeuva & $0,859 \mathrm{CD}$ & $1,75 \mathrm{~A} \mathrm{a}$ & $1,51 \mathrm{~A} \mathrm{a}$ & $1,67 \mathrm{~A} \mathrm{a}$
\end{tabular}

Em que: Valores seguidos de mesma letra maiúscula em uma mesma coluna não diferem estatisticamente entre espécie; e valores seguidos de mesma letra minúsculas em uma linha não diferem estatisticamente no decorrer do tempo, de acordo com o teste de Tukey a 5\% de significância.

Quando submetidos a campo de apodrecimento implantado em região nativa de Caatinga, o comportamento do processo de deterioração não foi afetado pela espécie (Tabela 4). Independente do tempo de exposição, não houve diferença significativa entre a perda de massa das espécies. Para a maioria das espécies, a deterioração também ocorreu dentro dos 90 dias iniciais, urundeuva $(0,859 \mathrm{~g} / \mathrm{cm} 3)$. Também não houve perda de massa significativa após os 90 e 200 primeiros dias de exposição. Apenas durante análise no dia 270 que foi observada diferença significativa entre a perda da espécie Anadenanthera columbrina (3,65\%) e as espécies Aspidosperma pyrifolium $(1,35 \%)$ e Myracrodrum urundeuva $(1,67 \%)$.

Tabela 4. Valores de perda de massa (\%) para as cinco espécies da Caatinga submetidas ao campo de apodrecimento natural após 90, 200 e 270 dias de exposição

\begin{tabular}{cccc}
\hline Espécies & 90 dias & 200 dias & 270 dias \\
\hline Anadenanthera colubrina & $1,03 \mathrm{~A} \mathrm{a}$ & $2,32 \mathrm{~A} \mathrm{a}$ & $2,36 \mathrm{~A} \mathrm{a}$ \\
Aspidosperma pyrifolium & $1,53 \mathrm{~A} \mathrm{a}$ & $2,69 \mathrm{~A} \mathrm{a}$ & $2,32 \mathrm{~A} \mathrm{a}$ \\
Auxemma oncocalyx & $0,80 \mathrm{~A} \mathrm{a}$ & $1,89 \mathrm{~A} \mathrm{ab}$ & $3,29 \mathrm{~A} \mathrm{~b}$ \\
Mimosa tenuiflora & $1,73 \mathrm{~A} \mathrm{a}$ & $2,52 \mathrm{~A} \mathrm{a}$ & $3,12 \mathrm{~A} \mathrm{a}$ \\
Myracrodruon urundeuva & $1,03 \mathrm{~A} \mathrm{a}$ & $1,71 \mathrm{~A} \mathrm{a}$ & $2,38 \mathrm{~A} \mathrm{a}$
\end{tabular}

Em que: Valores seguidos de mesma letra maiúscula em uma mesma coluna não diferem estatisticamente entre espécie; e valores seguidos de mesma letra minúsculas em uma linha não diferem estatisticamente no decorrer do tempo no teste de Tukey a 5\% de significância.

Todas as espécies avaliadas, independentemente do tipo de campo de apodrecimento ao qual foram submetidas, obtiveram valores médios de perda de massa inferior ao limite de $10 \%$ que as classifica com altamente resistentes de acordo com a norma americana (ASTM, 2005). Contudo, as perdas de massa em diferentes campos semelhante ao observado em campo aberto. Porém, uma espécie teve um aumento crescente ao passar do tempo, sendo ela a Auxemma oncocalyx. Neste caso isolado, o valor médio da perda de massa aos 270 dias de exposição $(3,29 \%)$ foi estatisticamente superior ao avaliado a 90 dias $(0,80 \%)$. 


\section{Carlos et al.}

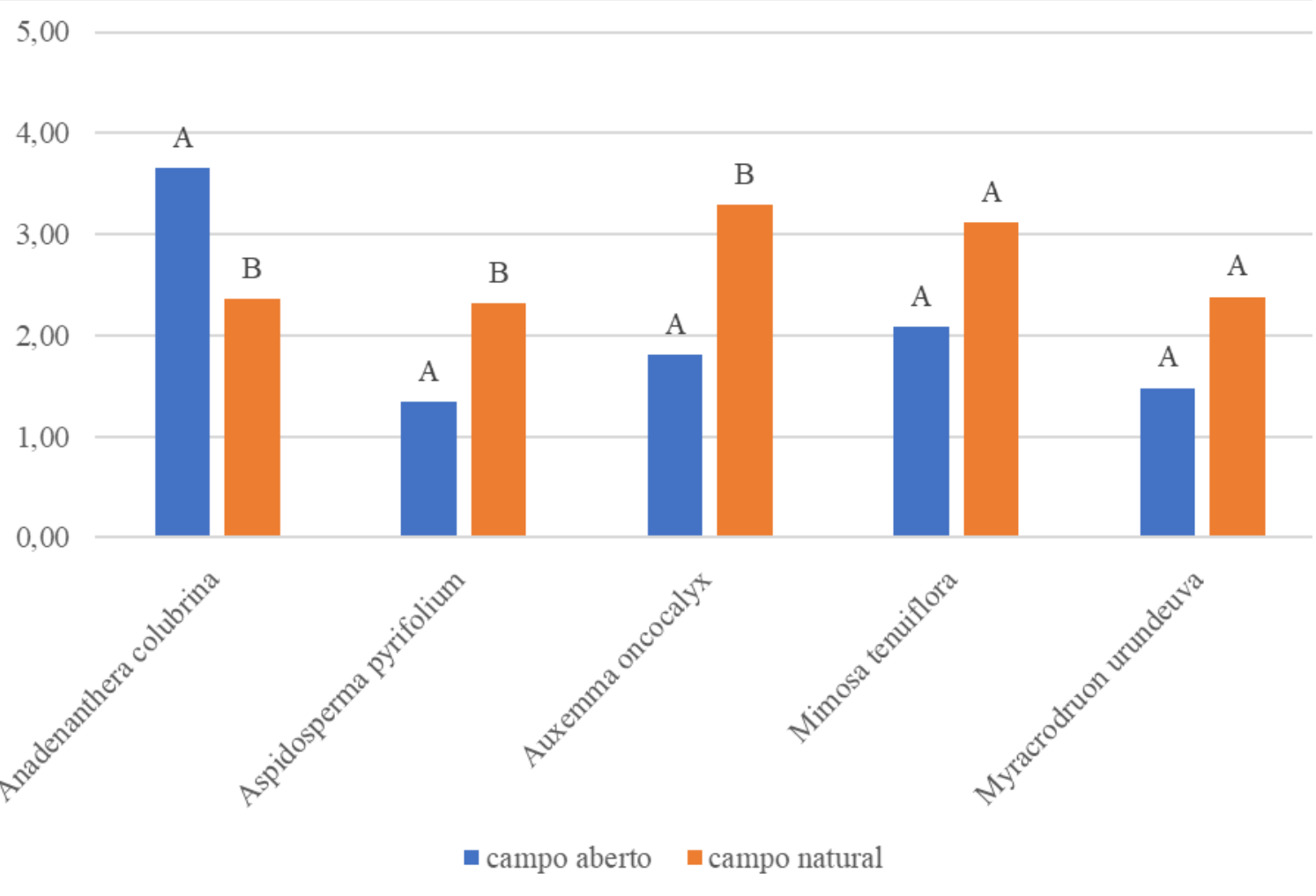

Figura 1. Comparação da perda de massa das espécies da Caatinga submetidas a campos de apodrecimento aberto e natural após os 270 dias de exposição. Colunas com letras iguais, na mesma espécie, não diferem estatisticamente, de acordo com o teste de Tukey a 5\% de significância.

Não houve diferença estatística entre os valores médios dos Índices de Deterioração das espécies estudadas após análise visual das estacas submetidas a 270 dias em ambos os tipos de campo (Tabela 5). Porém, mesmo que não tenha afetado a classificação, apenas duas espécies não apresentaram nenhum sinal de ataque de térmitas em nenhum dos campos durante todo o período de exposição: Aspidosperma pyrifolium e Auxemma oncocalyx. Em todos os ataques, os agentes biodeterioradores foram identificados como cupins subterrâneos do gênero Heterotermes. As espécies Anadenanthera colubrina e Myracrodruon urundeuva, além de ataque de cupins, também sofreu danos por coleópteras quando submetida ao campo aberto. No caso do ataque por coleópteras, não foi possível identificar o agente pois foi observado apenar o dano e não o indivíduo.

Tabela 5. Valores médio do índice de deterioração das diversas espécies da Caatinga submetidas a campo de apodrecimento aberto e natural

\begin{tabular}{ccc}
\hline \multirow{2}{*}{ Espécie } & \multicolumn{2}{c}{ Índice de Deterioração (\%) } \\
\cline { 2 - 3 } & Campo aberto & Campo natural \\
\hline Anadenanthera colubrina & $93,75 \mathrm{Aa}$ & $95,36 \mathrm{Aa}$ \\
Aspidosperma pyrifolium & $97,93 \mathrm{Aa}$ & $97,94 \mathrm{Aa}$ \\
Auxemma oncocalyx & $98,89 \mathrm{Aa}$ & $98,92 \mathrm{Aa}$ \\
Mimosa tenuiflora & $95,74 \mathrm{Aa}$ & $97,19 \mathrm{Aa}$ \\
Myracrodruon urundeuva & $95,55 \mathrm{Aa}$ & $95,89 \mathrm{Aa}$
\end{tabular}

Em que: Linhas seguidas de mesma letra maiúscula não diferem estatisticamente entre espécie; e colunas seguidas de mesma letra minúsculas não diferem estatisticamente no decorrer do tempo, de acordo com o teste de Tukey a $5 \%$ de significância. 


\section{Discussões}

O comportamento observado da predominância do ataque ocorrer nos primeiros 90 dias pode estar correlacionado com o período de instalação do experimento. As instalações dos campos ocorreram entre os meses de fevereiro e março de 2019 , período considerado chuvoso na região do município de Mossoró, RN. A partir do mês de julho, teve início o período de seca, com umidades relativas do ar inferiores a 70\% (Tabela 1). Teodorescu et al. (2017) determinaram ser possível prever a perda de massa por deterioração de qualquer madeira com modelos matemáticos que consideram a temperatura e umidade relativa do ar. De acordo com Michalski (1996), para preservar artefatos de madeira a umidade relativa ideal é abaixo de $60 \%$, porém o risco real de apodrecimento na maior parte dos casos ocorre somente acima de $75 \%$. A umidade relativa do ar média da região esteve abaixo desta faixa desde junho, quando ocorreu a primeira avaliação. Com pouca precipitação e baixa umidade relativa, as condições não foram ideias para o desenvolvimento dos fungos gerando uma estabilidade no grau de perda de massa.

Os valores médios encontrados para perda de massa em campo de apodrecimento aberto nas espécies Anadenanthera colubrina, Aspidosperma pyrifolium e Myracrodruon urundeuva foram semelhantes aos relatados por Paes et al. (2009) quando avaliaram madeiras de cerne destas espécies em campos simulados por 180 dias. Porém, quando estes autores simularam campo de floresta, valores médios apresentados para duas destas três espécies foram superiores aos observados neste experimento, apenas a madeira de Aspidosperma pyrifolium apresentou valores semelhantes.

A maior perda de massa apresentada pela madeira de Anadenanthera colubrina em relação às madeiras de Aspidosperma pyrifolium e Myracrodruon urundeuva, observado no campo de apodrecimento aberto pode estar relacionado a composição química das madeiras, contudo essa correlação não é direta ou simples. De acordo com Maia, Ferreira e Castro (2020), a espécie Anadenanthera colubrina possui valores de teor de extrativo solúveis em água quente e em acetona superior ao da espécie Aspidosperma pyrifolium. Porém, quando usado o solvente diclorometano, estas duas espécies apresentaram valores estatisticamente semelhantes. Como acetona é um solvente com alta capacidade de extração de componentes polares (Barbosa et al. 2005), pode ser confirmada a falta de correlação deste tipo de extrativo com a resistência da Anadenanthera colubrina, como indicada por Paes et al. (2009). Por outro lado, os mesmos autores correlacionaram a resistência natural da Myracrodruon urundeuva com o teor de extrativos em água quente. De fato, o teor de extrativo polar da Myracrodruon urundeuva foi de $18,73 \%$ (Paes et al. 2009), valor muito superior ao $6,18 \%$ da Anadenanthera colubrina (Maia, Ferreira e Castro, 2020), que poderia justificar a diferença da perda de massa entre as espécies neste experimento. Oliveira et al. (2005) apontaram que a resistência natural de uma espécie está realmente correlacionada com seu teor de extrativos, porém cada espécie produz seu extrativo específico que pode ser solúvel em diferentes solventes.

Ribeiro et al. (2014) comparando os resultados de campo de apodrecimento aberto e floresta utilizando-se madeira de Jequitibá (Cariniana micranta Duck) em período de seca observaram que a perda de massa foi maior no ambiente natural, semelhante ao comportamento apresentado pelas espécies Aspidosperma pyrifolium e Auxemma oncocalyx no presente estudo. A explicação apresentada pelos autores para aquele caso também pode ser aplicada aqui. Principalmente em regiões secas, o ambiente nativo apresenta características ecológicas inerentes que favorecem a atuação de organismos deterioradores, como uma cobertura vegetal que bloqueie a incidência direta de raios solares e capacidade de armazenar água no solo.

Em relação ao ataque de térmitas, Paes et al. (2013) observaram uma alta resistência da madeira de Anadenanthera colubrina a esse biodeteriorador em ensaio de preferência alimentar por 45 dias. Já Batista et al. (2020) apontaram uma alta resistência para a madeira de Myracrodruon urundeuva em condições de campo. A alta densidade das madeiras das espécies avaliadas pode ser o fator determinante para que, mesmo com indicativos da presença de térmitas, a perda de massa não tenha sido significativamente afetada. Madeiras de densidade elevada influenciam negativamente a capacidade dos cupins fragmentarem mecanicamente o material, o que dificultaria a perda de massa (Stallbaun et al. 2017).

\section{Conclusões}

As cinco espécies de madeiras com densidade moderadamente alta a alta da Caatinga são altamente resistentes aos ataques de biodeterioradores tanto em ambiente natural quanto em ambiente de campo aberto (em ambos, com valores de perda de massa abaixo de $4 \%$ ao final do experimento de 270 dias). Estas características em conjunto com as condições climáticas do semiárido brasileiro fazem com que estas espécies tenham um grande potencial de uso como matéria prima para construção civil ou indústria moveleira da região. É importante continuar pesquisas de caracterização química, física e mecânica destas espécies para que uma maior valoração seja agregada a este produto. 


\section{Referências}

Amorim IL, Sampaio EVSB, Araújo EL (2009) Fenologia de espécies lenhosas da Caatinga do Seridó, RN. Árvore, 33(3):491-499. doi: 10.1590/S0100-67622009000300011

Antongiovanni M, Venticinque, EM, Matsumoto M, Fonseca CR (2020) Chronic anthropogenic disturbance on Caatinga dry forest fragments. Journal of Applied Ecology, 57:2064-2074. doi:10.1111/1365-2664.13686

ASTM - American Society for Testing and Materials (2005) ASTM D-2017: standard method of accelerated laboratory test of natural decay resistance of woods. Philadelphia: ASTM. 5p.

Barbosa LCA, Maltha C, Cruz MP (2005). Composição química de extrativos lipofílicos e polares de madeira de Eucalyptus grandis. Ciência \& Engenharia (Science \& Engineering Journal), 15(2):13-20. doi: 10.1590/s1517-707620180004. 0560 .

Batista FG, Melo RR, Calegari L, Medeiros DT, Lopes PJG (2020). Resistência natural da madeira de seis espécies à Nasutitermes corniger Motsch. em condição de campo. Madera $Y$ bosques, 26(2):e2622017. doi:10.21829/myb.2020.2622017

Carvalho DE, Loiola PL (2018) Durabilidade natural. In: Castro VG, Guimarães PP (Org) Deterioração e preservação da madeira. Mossoró: EdUFERSA. p.125-134.

Carvalho DE, Santini EJ, Gouveia FN (2015). Resistência natural de quatro espécies florestais submetidas a ensaio com fungos apodrecedores. Floresta e Ambiente, 22(2):271-276. doi:10.1590/2179-8087.105914

Chaves EMF, Chaves EBF, Sérvio Júnior EM, Barros RFM (2014). Conhecimento tradicional: a cultura das cercas de madeira no Piauí, nordeste do Brasil. Etnobiología, 12(1):30-42.

Constantino R. (1999) Chave ilustrada para identificação dos gêneros de cupins (Insecta: Isoptera) que ocorrem no Brasil. Papéis Avulsos de Zoologia, 40(25):387-448.

Eleotério JR, Reichert D, Hornburg KF, Meneguelli I (2015) Massa específica e retratibilidade da madeira de seis espécies de Eucalipto cultivadas no litoral de Santa Catarina. Floresta, 45(2):329-336. doi:105380/rf.v45i2.34699

Ivanov MMM, Lacerda CF, Paiva FEF, Cavalcante ACR, Oliveira TS (2019) Environmental parameters and tree physiology in land use systems in Brazil. Journal of Forestry Research, 30(2):397-407. doi: 10.1007/s11676-018-0665-y

Lepage ES (1986) Manual de preservação de madeira. 2a Edição. São Paulo: IPT. 708p.

Lima JRF, Lima GDS, Lucena CM, Carvalho TKN, Lucena RFP (2018) Inventário in situ como método para avaliação da extração de recursos madeireiros na Caatinga: estudo de caso no município de Cabeceiras (Paraíba, Brasil). Revista Nordestina de Biologia, 26(1):1-18. doi:10.22478/ufpb.22361480v26n1.46051

Lopes EA, Canto JL (2018) Produtividade e custos de dois sistemas de exploração e transporte de lenha na Caatinga. Nativa, 6(2):207-212. doi: 10.31413/nativa.v6i2.4697

Lucena RFP, Farias DC, Carvalho TKN, Lucena $\mathrm{CM}$, Vasconcelos Neto CFA, Albuquerque UP (2011). Uso e conhecimento da aroeira (Myracrodruon urundeuva) por comunidades tradicionais no Semiárido brasileiro. Sitientibus série Ciências Biológicas 11(2):255-264. doi: $10.13102 / \mathrm{scb} 11$

Maia JH, Ferreira LMM, Castro VG (2020) Influence of extractives on the color of woods from Caatinga. Advances in Forestry Science, 7(2):10431048. doi:10.34062/afs.v7i2.9421

Mattos BD, Gatto DA, Cademartori PHG, Stangerlin DM, Beltrame R. (2013). Durabilidade a campo da madeira de três espécies de Eucalyptus tratadas por imersão simples. Revista Brasileira de Ciências Agrárias, 8(4):648-655. doi: 10.5039/ agraria.v8i4a3050

Michalski S (1996). Quantified risk reduction in the humidity dilemma. APT Bulletin: The Journal of Preservation Technology, 27(3): 25-29. doi:10.2307/1504412

Monteiro JM, Almeida CFCBR, Albuquerque UP, Lucena RFP, Florentino ATN, Oliveira RLC (2006) Use and traditional management of Anadenanthera colubrina (Vell.) Brenan in the semi-arid region of northeastern Brazil. Journal of Ethnobiology and Ethnomedicine, 2(6). doi:10.1186/1746-4269-2-6

Oliveira JTS, Souza LC, Lucia RMD, Souza Júnior P (2005) Influência dos extrativos na resistência ao apodrecimento de seis espécies de madeira. Revista Árvore, 29(5):819-826. doi: 10,1590/S010067622005000500017

Paes JB, Morais VM, Lima CR, Santos GJC (2009). Resistência natural de nove madeiras do semiárido 
brasileiro a fungos xilógafos em simuladores de campo. Revista. Árvore, 33(3):.511-520. doi:10.1590/S0100-67622009000300013

Paes JB, Medeiros Neto PN, Lima CR, Freitas MF, Diniz CEF (2013). Efeitos dos extrativos e cinzas na resistência natural de quatro madeiras a cupins xilófagos. Cerne, 19(3):399-405. doi: 10.1590/S0104-77602013000300006

Ribeiro MA, Stangerlin DM, Souza AP, Cardoso GV, Calegari L, Gatto DA (2014) Durabilidade natural da madeira de jequitibá em ensaios de deterioração em campo aberto e floresta durante as estações de seca e chuva. Comunicata Scientiae, 5(4):402-411. doi:10.14295/cs.v5i4.262

Sousa Júnior AD, Canto JL, Costa WPLB (2021) Produtos florestais nativos, legalizados e comercializados no Rio Grande do Norte. Cadernos de Ciência \& Tecnologia, 38(1):e26779. doi: 10.35977/0104-1096.cct2021.v38.26779

Souza GO, Pimenta AS, Trianoski R, Melo RR, Barbosa TK, Castro RVO (2020) Production of edge-glued panels with wood from three species and five types of adhesives. International Wood Products Journal, 11(2):57-63. doi: 10.1080/20426445.2020.1721041

Stallbaun PH, Barauna EEP, Paes JB, Ribeiro NC, Monteiro TC, Arantes MDC (2017). Resistência natural da madeira de Sclerolobium paniculatum Vogel a cupins em condições de laboratório. Floresta e Ambiente, 24(1): e20160013. doi: 10.1590/2179-8087.001316

Teodorescu I, Tapusi D, Erbasu R, Bastidas-Arteada E, Aoues Y (2017) Influence of the climatic changes on wood structures behaviour. Energy Procedia, 112(1):450-459. doi: 10.1016/ j.egypro.2017.03.1112 